\title{
Geometrically necessary dislocations and strain-gradient plasticity: a few critical issues
}

\author{
L.P. Kubin ${ }^{\mathrm{a}, *}$, A. Mortensen ${ }^{\mathrm{b}}$ \\ a Laboratoire d'Etude des Microstructures, CNRS-ONERA, 29 Av. de la Division Leclerc, BP 72, 92322 Châtillon Cedex, France \\ ${ }^{\mathrm{b}}$ Laboratoire de Métallurgie Mécanique, MX D 121, Institut des Matériaux, Ecole Polytechnique Fédérale de Lausanne, \\ CH-1015 Lausanne, Switzerland
}

Received 11 July 2002; accepted 9 August 2002

\begin{abstract}
A few issues related to the modeling of size effects in terms of geometrically necessary dislocations (GNDs) are critically discussed, viz. strain hardening, length scale dependence, types of GND arrays. Consequences are drawn regarding the continuum modeling of size effects in plasticity.

(c) 2002 Acta Materialia Inc. Published by Elsevier Science Ltd. All rights reserved.
\end{abstract}

Keywords: Dislocations; Constitutive equations; Theory and modeling — defects; Mechanical properties—plastic; Strain gradient plasticity

\section{Introduction}

One of the earliest mentions of a hardening effect due to the plastic accommodation of elastic strain gradients by dislocations is found in the book by Friedel [1]. With reference to the bending of a crystal to curvature $c$ (and with $\mu$ and $b$ being respectively the shear modulus and the modulus of the Burgers vector), Friedel wrote "... the minimum dislocation density necessary to produce the deformation is given by $\rho=c / b[\ldots]$. This density $[\ldots]$ introduces short-range stresses on a scale comparable with the average distance $\ell$ between dislocations $[\ldots]$. One expects therefore a para-

\footnotetext{
${ }^{*}$ Corresponding author. Tel.: +33-1-4673-4441; fax: +33-14673-4155.

E-mail address: kubin@onera.fr (L.P. Kubin).
}

bolic law $\sigma \approx \sigma_{0}+(\mu / 2 \pi)(b c)^{1 / 2}$. [...] Similar but more elaborate equations can be given in the same way for any type of macroscopic distortion which is not a uniform shear". These are of course geometrically necessary dislocations (GNDs) in the sense of Ashby [2], taken to induce increased hardening to the crystal in addition to that caused by statistically stored dislocations.

In pure fcc crystals, the resolved flow stress $\tau$ is then given by the well-known relationship:

$\tau=\alpha \mu b\left(\rho_{\mathrm{s}}+\rho_{\mathrm{g}}\right)^{1 / 2}$,

where $\alpha$ is a constant coefficient, $\rho_{\mathrm{s}}$ is the density of statistically stored dislocations and $\rho_{\mathrm{g}}$ the density of GNDs. With $\rho_{\mathrm{g}}$ proportional to the strain gradients, where Eq. (1) applies, GND-governed scale-dependent hardening is recognizable through a linear dependence of the square of the flow stress on the inverse of the distance $d$ characteristic of the 
deformation problem at hand (or, if $\rho_{\mathrm{s}} \ll \rho_{\mathrm{g}}$, a linear dependence of $\tau$ on $1 / \sqrt{d}$ ). Such a dependence has indeed been found in several important cases, including the response to microhardness indentations of fcc crystals, the large-strain flow stress of precipitate or dispersion hardened metals, and the yield and flow stress of particle reinforced metals [4-6].

GND models have attracted much attention because, in simple cases, the density of dislocations needed to relax a given strain gradient can be calculated assuming essentially static dislocation arrays dictated by local equilibrium. In a way, this is reminiscent of the low energy dislocation structure models [3], or of the models developed to explain the formation of arrays of misfit dislocations in epitaxial layers.

The resulting possibility of modeling scale effects in plastic deformation has motivated the proposal of several continuum plasticity theories which incorporate a dual dependence of the plastic flow stress on strain and strain gradients. The assumptions underlying these theories are variably based on the theory of GNDs, which is used to propose length scales that serve to quantify in the continuum the contribution of strain gradients to hardening. Our purpose in this short contribution is to discuss, from a materials science perspective, a few critical issues in connection with the dislocation phenomena that underlie the continuum strain gradient plasticity (SGP) approach.

\section{Hardening by geometrically necessary disloca- tions}

How valid is Eq. (1)? In pure fcc metals, it is quite robust. The flow stress is then mostly governed by short-range attractive intersections of non-coplanar dislocations. As was shown by early theoretical studies $[7,8]$, and confirmed by experimental investigations [9], this leads to Eq. (1) with $\alpha=0.3 \pm 0.1$. This "forest" hardening should be distinguished from "dipolar" or Taylor hardening which stems from long-range dislocation interactions. Then, the same scaling law is recovered but the corresponding value of the coefficient $\alpha$ critically depends on specific hypotheses made re- garding the GND spatial distribution. This applies to situations where GNDs are assumed to be stored at an interface, for instance a grain boundary in a polycrystal or a phase boundary in a composite material, and cannot play the role of forest obstacles.

A more subtle question concerning Eq. (1) in fcc metals is that of the distinction between statistical and geometrically necessary dislocations. In a recent study of the deformation of bulk aluminum polycrystals, Hansen and Huang [10] have succeeded in estimating the density of GNDs from TEM measurements. The dislocation microstructures are made up of subgrains or walls, which accommodate the misorientations between dislocation-poor areas. From direct measurements of the local misorientations, the density of dislocations within cell walls was deduced and the coefficient $\alpha$ was estimated from the knowledge of a Taylor-averaged flow stress. Its value decreases from 0.28 to 0.2 for strains increasing from $5 \%$ to $34 \%$. This agrees relatively well with theoretical expectations (within a proper treatment of line tension effects this coefficient decreases slowly with increasing dislocation density), as well as experimental [9] or simulated [11] values. The interesting point is that, in this case, the majority of the dislocations are in the walls, and hence are GNDs accommodating naturally occurring spatial strain variations. As far as the hardening behavior is concerned they behave in the same manner as statistically stored dislocations, to which they are implicitely assimilated in SGP. These GNDs actually are at the origin of most of the flow stress of the material at large strains $\left(\rho_{\mathrm{g}} \gg \rho_{\mathrm{s}}\right)$, whereas $\rho_{\mathrm{g}}$ should be negligible with respect to $\rho_{\mathrm{s}}$ at small strains, since local misorientations are very small. Unfortunately, very little is know about the strain dependencies of these two densities when they are considered separately.

As soon as one leaves pure fcc metals, the validity of Eq. (1) comes into question. In fcc crystals, if dislocation motion requires overcoming a friction stress $\tau_{0}$ (for example because of solid solution strengthening) it is conventionally accepted to simply add this stress to the right-hand side of Eq. (1); SGP equations were recently adapted to account for this [12]. In materials other than fcc 
crystals, in particular those where the flow stress is not governed by forest interactions, both Eq. (1) and its modification with a constant friction stress can be invalid. This includes for instance bcc metals or transition hep metals like Ti or Zr, where the dislocation mobility is reduced by a strong lattice friction due to the non-planar configuration of the screw dislocation cores. In such cases, strain hardening stems from a complex superposition between forest hardening and lattice friction [13], leading to a relation between flow stress and total density that is more complex than Eq. (1) and no longer involves a square root relationship. Eq. (1) then applies only when the lattice friction stress (or Peierls stress) is significantly smaller than forest hardening, i.e., when it is reduced by thermal activation at high temperatures and low strain rates, or at large strains and dislocation densities such that forest hardening prevails. This difficulty is not recognized in many situations involving bec crystals. Then, one simply cannot assume additivity of the lattice friction and of forest hardening, for instance when modeling the indentation size effect in tungsten at room temperature [12]. Indeed, lattice friction governs plastic flow up to about $600 \mathrm{~K}$ in this material. For other bcc crystals a very rough rule of thumb involves scaling this temperature either by $\mu b^{3}$ or by the melting temperature.

\section{Length scale dependence}

As mentioned in the previous section, the observed dependence of hardening on length scale predicted by Eq. (1) has, indeed, been observed in several important cases. This provides strong justification for the use of GND theory towards quantification of scale-dependent plasticity; however, as is well known, this dependence is not universal. In particular, the initial yield stress of undeformed materials, which at a fine scale of structures is also scale-dependent, generally cannot be explained by GND theory, simply because generally there is no GND accumulation before yield (an exception is provided by metal matrix composites, which are hardened before tensile yield by GNDs of thermal origin).
Despite the fact that it displays a linear dependence of flow stress on the inverse square root of the characteristic distance $d$, the Hall-Petch yield stress of annealed metals is thus not GND-governed. This size effect is traditionally modeled in terms of dislocation pile-ups at grain boundaries, whereas a model of GND storage is used to describe polycristal strain hardening [5]; both lead to the same scaling. This switching between very different types of configurations to describe the same scaling behavior may be found a bit puzzling in conceptual terms.

Yield stresses also often display different functional dependencies on $d$, which complicates modeling. For example, with shearable precipitates, the yield stress increases with $d$, following a variety of laws which depend on the specifics of precipitate shear [14]. Frequently, yield is directly governed by Orowan bowing to a critical radius that scales directly with $d$ (yielding between non-shearable precipitates or dispersoids, narrowly confined plasticity in thin films or between hard second phases, ...). As a consequence, the scale-dependent increment in yield stress depends linearly on $(1 / d)$, or more correctly on $(\ln (d) / d)$. For instance, in the deformation of polycrystalline copper thin films on a substrate [15], the yield stress scales as $1 / d$ due to confinement, whereas the strain hardening behavior does not and may be qualitatively understood in terms of GND storage. Confinement size effects are also currently observed in lamellar materials and in superalloys with large volume fractions of the hard phase, where the width of the ductile channels is about $0.1 \mu \mathrm{m}$. Considering their practical importance, it is remarkable that confinement effects in such materials have only very recently been modeled in the continuum [16].

In all these cases, the functional relation between length scale and flow stress cannot be modeled from SGP analyses based on parabolic dislocation hardening according to Eq. (1). Generally, $d$-values at which direct Orowan or other non-GND size-dependencies of the yield stress become noticeable are roughly at and below $1 \mu \mathrm{m}$. This range overlaps partly with that in which SGP models are supposed to apply.

GND theory also assumes that dislocation nucleation proceeds unhindered. This is not always 
true, particularly at small length-scales. Precipitates or second phase particles, for example, will begin to encounter difficulty in nucleating GND loops when their size falls below a certain value in the rough vicinity of $0.1 \mu \mathrm{m}$ : clearly, then, the theory cannot be applied. Phenomena such that dislocation nucleation has to occur in small, initially dislocation-free, volumes place a lower limit on the range of $d$ values over which GND-based strain-gradient plasticity theory may be useful. In some cases, for instance in nanostructured materials, this may narrow significantly the range of applicability of the theory.

\section{Specific nature of geometrically necessary dislo- cation patterns}

As pointed out by Gil Sevillano: "GND arrays are not univocally determined" [17]. This is well illustrated by the variety of dislocation configurations that exist in a system as basic as a crystal deforming by single slip around a hard spherical particle $[5,18]$.

As an illustration of the importance of this point, consider the SGP model proposed by Gao et al. [19]. A salient feature of this model is its clear concern for basing the SGP equations on specific GND arrays, which it describes in four cases. One is bending: in this case the array of parallel edge dislocations lends itself as the obvious solution. The second case is simple torsion of a cylinder: the authors propose a series of screw dislocations coaxial with the cylinder as the relevant array of GNDs. A likely alternative (as in all such simple models, in a well oriented crystal) is that of a series of twist subgrain boundaries in the cylinder, containing regularly spaced parallel arrays of screw dislocations oriented normal to the axis. Assuming for simplicity that each subboundary contains two perpendicular arrays of screw dislocations, the dislocation density required to accommodate a twist angle $\kappa$ over unit length is easily shown to be

$\rho_{\mathrm{G}}=2 \kappa / b$,

i.e., twice the density assumed in [19].

The third case considered is that of cylindrically symmetric outward expansion, corresponding to the growth of a cylindrical void or inclusion in a crystal. Here the GND array considered by Gao et al. consists of concentric crowns of edge dislocations having their axis parallel to the void, and their Burgers vector oriented along the $\theta \theta$ direction (cf. [19, Fig. 3(c)]). A more realistic GND array is that which is actually observed in thermal mismatch strain relief by fibers [20]. It consists of long prismatic loops having their Burgers vector oriented along slip directions closest to the radial direction in the problem at hand. Assuming these long prismatic dislocation loops have a width of $\sqrt{2}$ times the void/fiber radius, $r_{0}, \sqrt{2} \pi u_{0} / b$ loops are required to relieve the mismatch volume created by a surface displacement $u_{0}$ at the surface of the void/inclusion. The resulting total dislocation length per unit cylinder length is then:

$L=2 \sqrt{2} \pi u_{0} / b$,

which is lower by a factor $\sqrt{2}$ than the value derived from the configuration assumed in [19].

In the fourth case considered by Gao et al., namely spherically symmetric outward expansion at the surface of a spherical hole or inclusion, another GND array, similar to the previous one, can be proposed. Indeed, it is well known that circular prismatic loops with their Burgers vector oriented along the radial direction relieve the mismatch strain in such cases. Here, the total prismatic dislocation loop length required is

$L=8 \sqrt{2} \pi r_{0} u_{0} / b$,

which is again lower, by a factor of $\sqrt{2} / 3$, than is assumed in [19].

Assumed GND arrays are used in the theory to estimate a set of three constants $\left(c_{1}, c_{2}, c_{3}\right)$, from which the strain gradient tensor is defined (cf. [21; 19, Eq. (23)]). With the above distributions, this set becomes $(-11 / 24,17 / 24,-14 / 24)$ instead of $(0,1 / 4$, 0 ) in the original model, assuming the same three cases and the same strain distributions in the calculation of these constants (this is a simplification given the more complex local strain distribution associated with the above GND patterns). Other permutations of these cases and their chosen GND arrays could be used to obtain other sets of values. The implications are (i) that the assumed GND 
configurations matter significantly in defining the SGP law assumed, and (ii) that, reciprocally, any given SGP model carries implicitely in it the assumption of a certain rather precisely defined GND array configuration, which may be at variance with reality in the different cases where the model is applied. This is a fundamental limitation of SGP models, which results from their assumption that one to three scalars can fully render the influence of relatively complex "real" GND arrays. In reality, GND arrays are governed by a combination of energetic, crystallographic, and kinetic phenomena, which often influence significantly the final GND distribution and density. The cases of spherical or cylindrical inclusions considered above are, again, interesting in this regard. If prismatic loops do not get entangled near an inclusion, they can glide over considerable distances, creating extended patterns which do not reflect the symmetry of the inclusion. This is something no present continuum SGP model can capture.

We now return to Eq. (1), noticing that it is expressed in terms of spatially averaged dislocation densities and nevertheless yields good results in the case where the dislocation microstructure is self-organized (cf. [10] and Section 2). This rather striking feature is not understood. Tentative explanations have been proposed, for dislocation cell structures, in terms of Mughrabi's "composite model" [22] and in terms of the "forest model" by Neuhaus and Schwink [23]. Because of this relative insensitivity to the spatial arrangement of the microstructure, size effects induced by externally imposed strain gradients, e.g., in bending or torsion, can effectively be modelled in terms of a known total density of GNDs. It is not so, however, for patterned dislocation structures that spontaneously form in conditions where no strain gradient is imposed. In monotonic deformation, these microstructures can schematically be described as containing subgrains of micrometer dimensions, with small alternating misorientations, and larger subgrains or cell-blocks accommodating large misorientations (cf. e.g., [24]). Our present level of understanding does not allow to predict the two corresponding characteristic scales for these "natural" strain gradients, nor how the misorientations grow with increasing strain. Fur- ther, the average strain gradient inside a single cell block is close to zero, due to the alternance of misorientations at smaller scale. In short, it is not presently possible to propose convincing models, either discrete or continuum, of dislocation patterning in terms of GNDs.

\section{Gradient plasticity and size effects}

Several difficulties arise when formulating continuum models for the evolution of dislocation densities inside a crystal. We discuss here the homogenization process that transforms a discrete dislocation density into a continuum one, within a purely dislocational framework and without making reference to GNDs. In addition we show that the modeling of size effects of dislocational origin does not necessarily involves the consideration of GNDs.

Models describing the coupled evolution of dislocation populations in time $(t)$ and space have been initiated by Walgraef and Aifantis [25] and were further developed in the past years in the context of dislocation patterning. A balance equation is written within a small homogenization volume of linear dimension $\ell$. In scalar terms and for each population, we have:

$\partial \rho / \partial t+\operatorname{div}(\rho v)=$ reactions

where $v$ is a dislocation velocity and $\rho v$ a dislocation flux. The reaction side may include a number of dislocation mechanisms (multiplication, annihilation, blocking, etc.).

An example of an annihilation mechanism is shown in Fig. 1, namely the annihilation by crossslip of two screw dislocations of opposite sign. Under the effect of their mutual attraction, the two segments can move out of their slip plane and annihilate provided that the distance between their slip planes is smaller than a critical, stress-dependent, value $h_{\mathrm{c}}$. We see from Fig. 1 that if $\ell$ is taken smaller than $h_{\mathrm{c}}$, cross-slip enters the transport term at the left-hand side of Eq. (1), leading to a gradient form once the flux term is expanded. Conversely, if $h_{\mathrm{c}}<\ell$, the annihilation process is a reaction term and its spatial aspects are averaged 

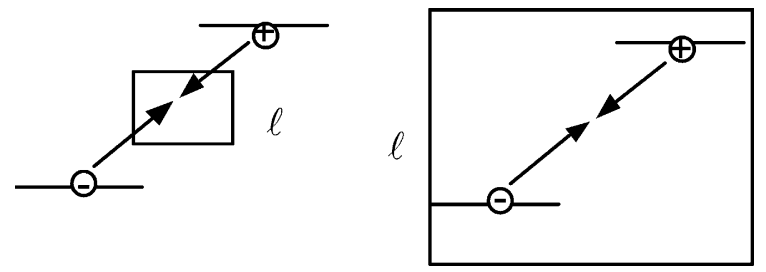

Fig. 1. Homogenization of a dislocation process, here the annihilation of two screw dislocations of opposite sign, with different coarse-graining dimensions.

out. More generally, a length scale can be attached to each dislocation mechanism, thus defining a hierarchy of length scales. The related mechanisms are for instance the annihilation of edge dipoles, the average distance between dislocations or between other obstacles, the typical width of cell walls, cell diameters and the grain size in a polycrystal. The related length scales may, in addition, depend on stress (cf. Section 3). If $\ell$ increases, mechanisms are progressively shifted from the transport-side to the reaction-side of Eq. (1), until $\ell$ becomes of the order of the specimen size. Then, the whole dislocation density is spatially averaged. When $\ell$ decreases, more mechanisms appear on the transport side of Eq. (1), until the homogenization volume contains either zero or one dislocation. At this point, the model becomes discrete.

In fact, the dimension of the homogenization volume cannot be fixed by a physical argument. It is somewhat arbitrary and depends upon the nature of the mechanisms and length scales that one wishes to account for in a given model. Thus, there are many possible models and the homogenization length scale $\ell$ governs their spatial resolution, although it never appears explicitly in Eq. (5). It seems that the occurrence of this spatial dividing line is the price to pay for integrating the inherently discrete character of dislocation mechanisms into a continuum description.

\section{Concluding remarks}

GND models can efficiently be used to describe size effects in plasticity, but within some limits, however. These limits are set by the static and equilibrium characters of the GND framework, by kinetic effects such as lattice friction and by yield phenomena also, which may exhibit different, nonGND related, scaling properties at micron-scale distances. Other limitations stem from the importance of GND array specifics or, in other words, by the potential lack of universality of characteristic lengths used for comparison with strain gradients. We believe these limitations will cause continuum SGP models to face, in the near future, a serious challenge from truly multiscale approaches, which combine an atomic-scale treatment of nanosized configurations with simulation methods for dislocation effects at the meso-scale, together with a capacity to tackle sophisticated boundary value problems (e.g., via phase field methods or coupled finite elements and dislocation dynamics simulations).

\section{References}

[1] Friedel J. Dislocations. Oxford: Pergamon Press; 1964. p. 254.

[2] Ashby MF. Phil Mag 1970;21:399.

[3] Kuhlmann-Wilsdorf D. Mater Sci Eng A 1989;113:1.

[4] Nix WD, Gao H. J Mech Phys Solids 1998;46:411.

[5] Ashby MF. In: Kelly A, Nicholson RB, editors. Strengthening methods in crystals. Amsterdam: Elsevier; 1971. p. 137.

[6] Kouzeli M, Mortensen A. Acta Mater 2002;50:39.

[7] Saada G. Acta Metall 1960;8:841.

[8] Schoeck G, Frydman R. Phys Stat Sol B 1972;53:661.

[9] Basinski SJ, Basinski ZJ. In: Nabarro FRN, editor. Dislocations in solids, vol. 4. Amsterdam: North Holland; 1979. p. 261.

[10] Hansen N, Huang X. Acta Mater 1998;46:1827.

[11] Madec R. Doctoral dissertation. University Paris XI, 2001.

[12] Qiu X, Huang Y, Nix WD, Hwang KC, Gao H. Acta Mater 2001;49:3949.

[13] Tang M, Devincre B, Kubin LP. Modell Simul Mater Sci Eng 1999;7:753.

[14] Reppish B. In: Cahn RW, Haasen P, Kramer EJ, editors. Plastic deformation and fracture of materials. Mughrabi H, editors. Materials science and technology, vol. 6. 1993. p. 311 .

[15] Hommel M, Kraft O. Acta Mater 2001;49:3935.

[16] Forest S, Sedlacek R. Phys Stat Sol B 2000;221:583.

[17] Gil Sevillano J. In: Kubin LP, Selinger RL, Bassani JL, Cho KJ, editors. Multiscale modeling of materials - 2000, Symposium Proceedings, vol. 653. Warrendale, PA: Materials Research Society; 2001. p. Z8.5. 
[18] Brown LM. In: Haasen P, Gerold V, Kostroz G, editors. Strength of metals and alloys, Proceedings of ICSMA 5. Oxford: Pergamon Press; 1980. p. 1551.

[19] Gao H, Huang Y, Nix WD, Hutchinson JW. J Mech Phys Solids 1999;47:1239.

[20] Dunand DC, Mortensen A. Acta Metall Mater 1991; 39:127, 1417.
[21] Fleck NA, Hutchinson JW. J Mech Phys Solids 1997; 41:1825.

[22] Mughrabi H. Mater Sci Eng 1985:15-31.

[23] Neuhaus R, Schwink Ch. Phil Mag A 1992;65:1463.

[24] Bay B, Hansen N, Hughes DA, Kuhlmann-Wilsdorf D. Acta Metall Mater 1992;40:205.

[25] Walgraef D, Aifantis EC. J Appl Phys 1985;58:688. 